\title{
Simple Mechanics to Upright Horizontally Impacted Molars with Ramus Screws
}

\author{
Dr Shih-Yung Lin,' Dr Chris Chang,2 W. Eugene Roberts \\ "BOI Diplomate, ${ }^{2} F o u n d e r$, Beethoven Orthodontic Center, Publisher, International Journal of Orthodontics \& Implantology, \\ ${ }^{3}$ Consultant, International Journal of Orthodontics \& Implantology
}

\section{ABSTRACT}

Simplified mechanics are reported for uprighting horizontally impacted mandibular molars with ramus bone screws. A 27 -yearold male presented with a chief complaint of food impaction and problematic occlusion of his posterior teeth. The panoramic radiograph revealed multiple impacted molars: both upper third molars, and all lower second and third molars. Orthodontic treatment was indicated to improve occlusal function by aligning the posterior segments. The treatment plan was to extract the upper second molars and lower third molars, then upright the lower second molars, and allow the upper third molars to spontaneously erupt. The deeply impacted lower second molars were uprighted in only $\sim 4$ months with superiorly positioned ramus bone screws.

Key words: horizontally impacted second molars, molar uprighting, ramus bone screws

\section{INTRODUCTION}

Impaction risk is greatest for mandibular and maxillary third molars, followed by maxillary canines and mandibular second molars.' Depending on the sample, the prevalence of impacted second molars is up to $2.3 \% .^{2,3}$ The etiology of impaction may be ectopic position of the third molar, obstacles in the eruption path, and failure of the tooth eruption mechanism. ${ }^{4}$ This case report demonstrates simple

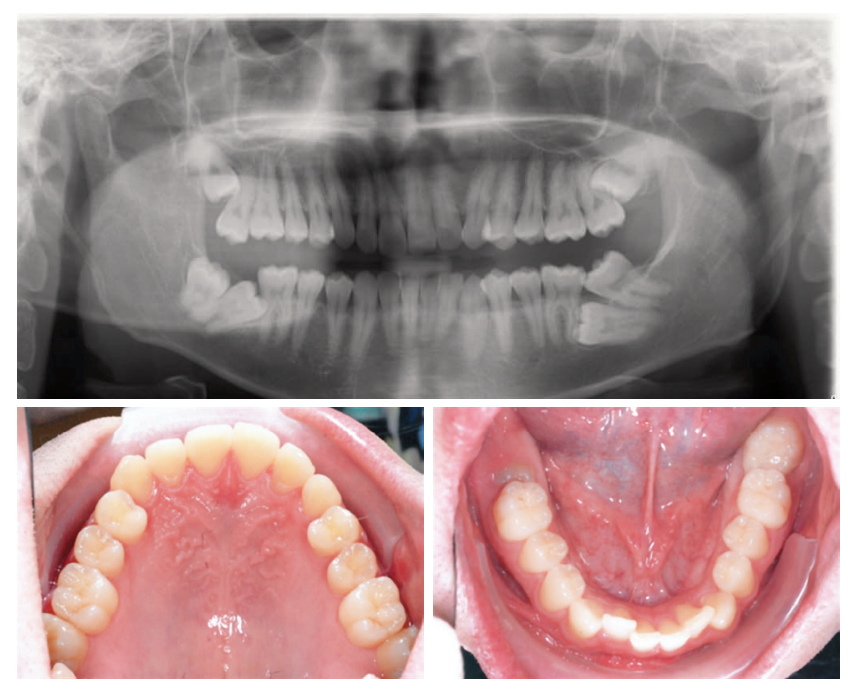

Figure 1: Pre-treatment panoramic radiograph (above) \& occlusal intra-oral photographs (below) document the malocclusion. mechanics for managing horizontally impacted second molars in $\sim 4$ months. Detailed, step by step procedures are reported.

\section{CASE REPORT}

A 27-year-old male presented with bilaterally deeply impacted lower second molars, and a mesially tipped lower left third molar. The other third molars were impacted (Figure 1).

\section{TREATMENT PLAN OPTIONS}

Upper Arch

1. No treatment is not a viable option because it fails to address the functional problems, and the impacted third molars may result in root resorption of the adjacent second molars, as well as cyst formation and/or referred pain. ${ }^{5}$

2. Extract the impacted third molars and align the second molars. It may be difficult to extract the third molars without damaging the second molars. Furthermore the extraction site may heal with a soft tissue defect on the distal of the second molars.

3. Remove the second molars and allow for spontaneous eruption of the third molars. After spontaneous eruption of the thirds molars, the buccal segments can be aligned 


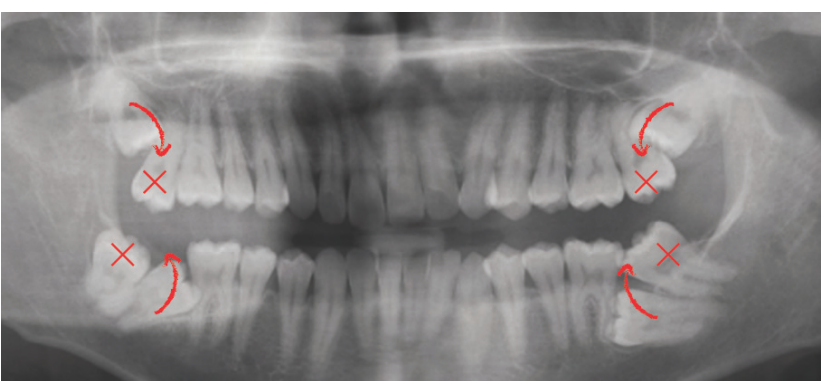

Figure 2: The treatment plan was to extract the molars marked with an $\mathrm{X}$, upright the lower second molars, and allow the upper third molars to erupt.

with fixed appliances. Extraction of the erupted second molars presents less surgical risk than removing the impacted third molars.

\section{Lower Arch}

1. Remove all second and third molars, place a subperiosteal membrane for guided bone regeneration, and place implants to restore the second molars. The major concern with this option is the possibility of the inferior alveolar nerve injury during the extraction process, and healing of the surgical site is unpredictable.

2. Remove the impacted third molars, and then upright the second molars with elastic chains anchored by bone screws placed in the ramus, bilaterally.

After careful discussion of the options with the patient, the decision was made to extract the upper second molars and the lower third molars (Figure 2), and place bilateral ramus bone screws (Figure 3). Attachments were bonded on the distal surface of each second molar (Figure 4).

\section{SURGICAL PROCEDURES}

Under local anesthesia, bone screws $(2 \times 14 \mathrm{~mm}$ stainless steel) were inserted in the ramus of the mandible bilaterally (Figure 4). Then, the upper second and lower third molars were extracted, followed by surgical exposure of the lower second molars. An explorer was used to locate the crowns of impactions. Teeth are smooth and hard, while bone is crunchy and irregular.

Bone superior to the lower second molar crowns was removed down to the level of the cementoenamel junction (CEJ) with a high-speed handpiece, The second molars were surgically luxated to rule out ankylosis. A button was bonded on the distal surface of the lower right second molar, and an eyelet was bonded on the left second molar (Figure 4).

Elastic chains, anchored by the bone screws, were activated by connecting the opposite end of chain to the attachments bonded on the second molars (Figure 5). A resin ball was applied to the bone screw head to prevent the elastic chains from being displaced (Figure 4). To control bleeding, the soft tissue wound was closed with interrupted sutures which were removed after one week. Four months later the second molars were uprighted successfully and routine brackets were bonded on the buccal surface at five months into treatment (Figure 5).

\section{DISCUSSION}

Deciding between extraction or uprighting for impacted teeth is a critical aspect of treatment planning. Factors affecting the decision are severity of the impaction, its position relative to critical anatomical structures (inferior alveolar nerve

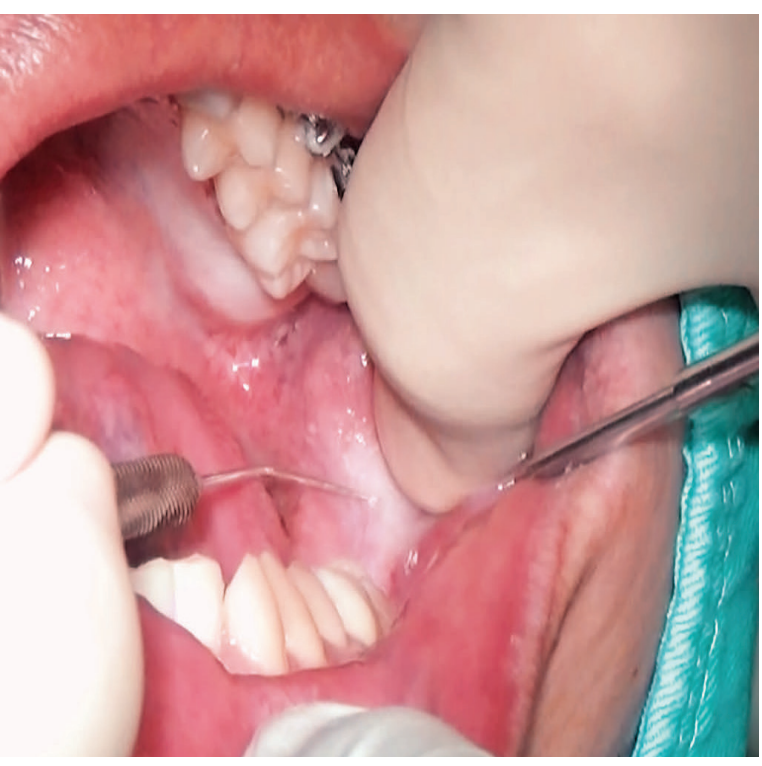

Figure 3: Under local anesthesia, the insertion site on the left ramus is marked with an explorer by penetrating the soft tissue to the bone.
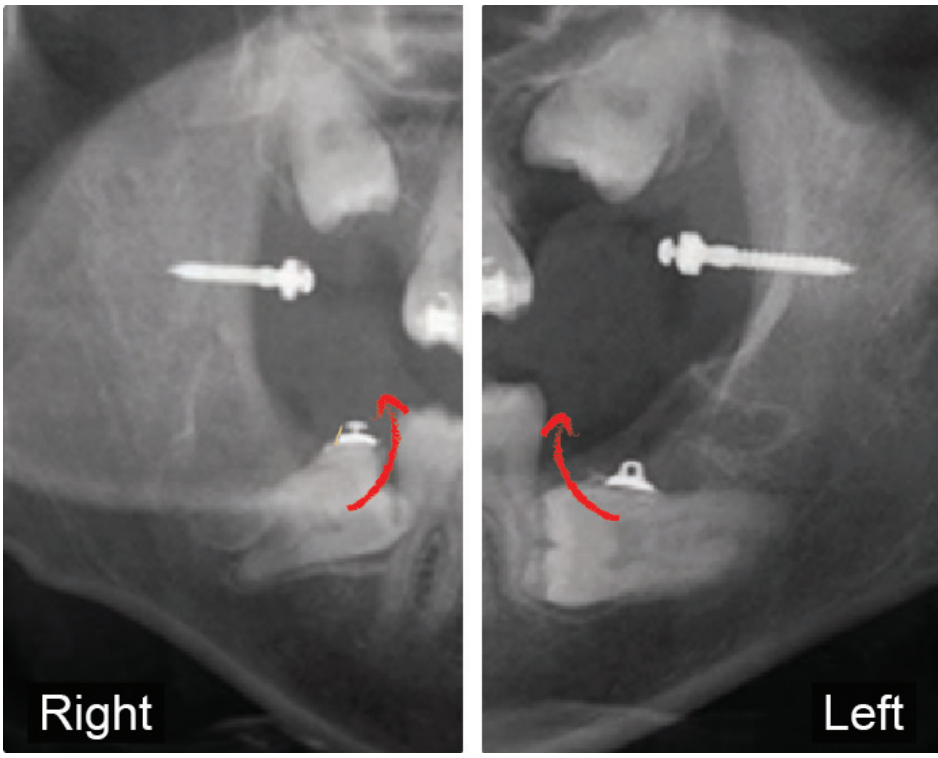

Figure 4: The right and left ramus areas are shown in segments of a postoperative panoramic film taken immediately after surgery. A button was bonded on the right side and an eyelet was used on the left side. 

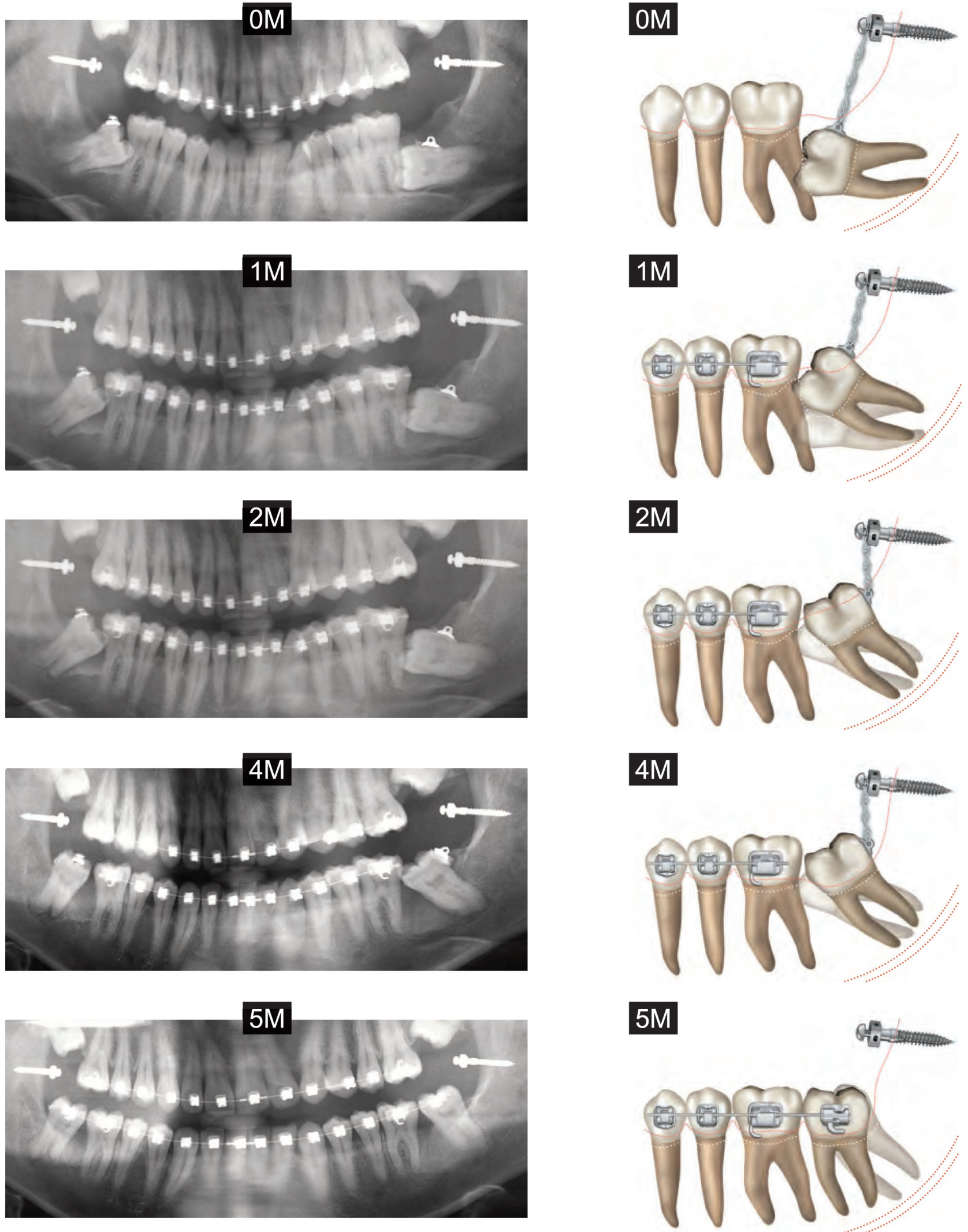

Figure 5: On the left, a series of panoramic films document tooth positions immediately after surgery $(0 \mathrm{~m})$, then at $1(1 \mathrm{~m}), 2(2 \mathrm{~m}), 4(4 \mathrm{~m})$ and 5 months $(5 \mathrm{~m})$ later. Corresponding drawings documenting the mechanics are shown on the right. 


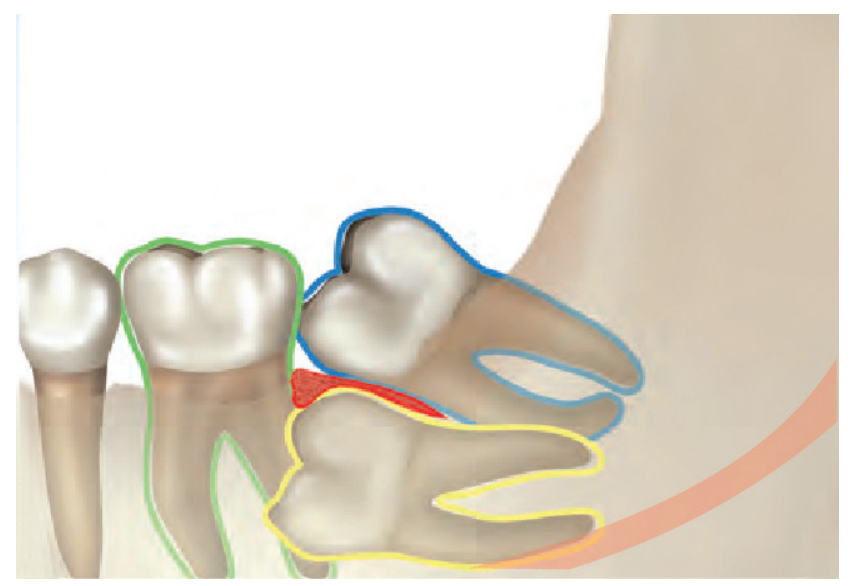

Figure 6: The etiology for an impacted second molar may be a superiorly positioned third molar (blue), dense bone blocking eruption (red), ankylosis of the second molar (yellow), and the undercut of the adjacent first molar (green). The inferior alveolar canal (pink) is very close to the root of the impacted second molar.

and lingual artery etc), pre-existing conditions of impacted teeth (caries, root dilaceration, periodontal health etc), and difficulty of the extraction surgery (Figure 6). If orthodontics is indicated, an efficient mechanics plan is required.

Many methods of uprighting molars have been proposed in the literature.6 The mechanical design presented here is simple, effective and expedient. There are two essential tips to ensure routine success with the proposed method.

Tips 1. Remove all obstructions to eruption, such as ectopic position of the dental follicle, teeth or pathology in the path of eruption, and failure of the tooth eruption mechanism.? For the present patient, the suspected etiology involves the following (Figure 6):

a. Impacted third molar putting pressure on the second molar

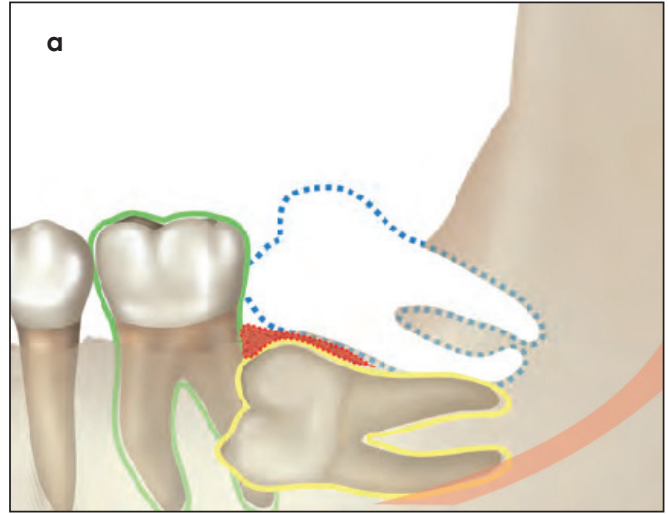

Figure 7(a) : After extraction of the lower third molar (blue), the covering bone (red) was removed with a high-speed handpiece. b. Thick cortical bone blocking the eruption of the second molar

c. Ankylosis of the second molar, and/or

d. Ectopic eruption of the second molar to engage the undercut on the distal of the first molar.

For predictable uprighting and alignment of the second molars, all of the potential etiologic factors were addressed by extracting the third molars and gently luxating the second molars (Figure 7).

Tips 2. Design an efficient force system for uprighting the impacted teeth. 8.9 Uprighting a deep horizontal impaction requires occlusal and distal components of force to unlock the impaction from its position against the first molar root. It is wise to avoid bonding a bracket on the first molar until the second molar is at least partially uprighted. This will allow the first molar to move out of the way to help avoid root resorption. Miniscrews can provide favorable anchorage without side effects on the dentition. The dense cortical bone in the ramus is a good site to insert the screw (Figure 7).

Furthermore, there are several considerations in selecting bonding devices and bonding sites. The most popular bondable attachments for uprighting molars are buttons or eyelets (Figure 8). An eyelet is more versatile, but it more difficult to use, compared to a button. In deciding between the two options, the first concern is the line of force. Buttons are well designed for horizontal traction (Figure 8), but elastic chains are more easily displaced as the direction of traction assumes a more vertical orientation. The second consideration is the convenience of placing and replacing the elastic chains. An eyelet is easily bonded on a tooth with the elastic attached, but a button is more convenient if the elastic must be changed (Table 1). If the attachment is bonded on the surface of the erupted tooth, and the angle of traction is appropriate (Figure 9), a button may be the preferred choice.

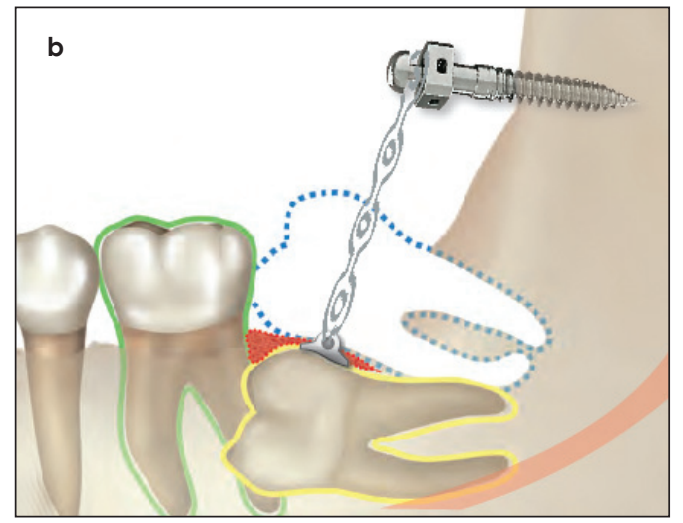

Figure $7(b)$ : The ramus screw was inserted into the ascending ramus, and an elastic (power) chain with a line of force occlusal to the center of resistance was used to upright the horizontally impacted second molar. 
Table 1: Bondable attachments, buttons and eyelets, are compared relative to clinical efficiency

\begin{tabular}{|l|l|l|}
\hline & \multicolumn{1}{|c|}{ Button } & \multicolumn{1}{c|}{ Eyelet } \\
\hline Traction direction & Parallel / Slightly angular & Any direction \\
\hline Replacing elastic & Simple and easy & More complex \\
\hline Method o operation & Difficult & Easy \\
\hline
\end{tabular}

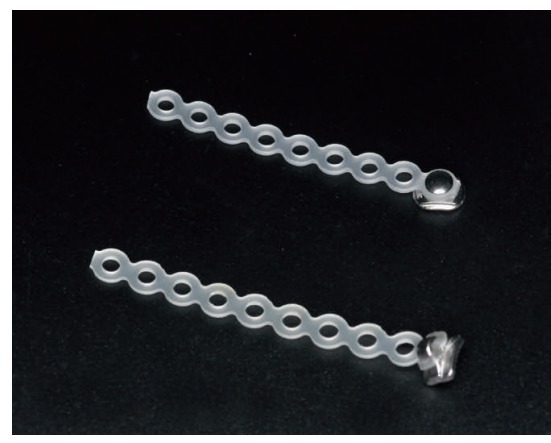

Figure 8: Bondable button (above) and eyelet (below) attachments are fitted with elastic chains.
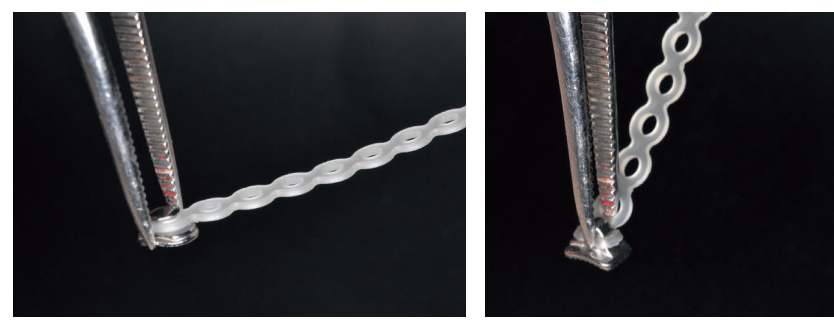

Figure 10: A button with an elastic chain attached (left) is difficult to hold securely. In contrast, the flat surfaces of an eyelet facilitate securely holding it with a hemostat.

However, if the attachment is bonded on the surface of an unerupted tooth, an eyelet is preferable because there is less chance that the elastic chain will come loose. Flowable resin can be polymerized to secure the elastic chain on either type of attachment. An additional determinant is the convenience when holding the device with a hemostat or pliers. A button has a smooth, rounded head so it is more difficult to hold. The flat surfaces of an eyelet are more amenable to holding the button for bonding or any other manipulation (Figure 10).

\section{Why Ramus Screws?}

An efficient, yet simple mechanism is required to upright deeply impacted or mesially tipped molars. Lin6 reviewed six different methods of molar uprighting, and concluded that surgical exposure of the deeply impacted molars, followed by traction with elastic chains anchored by ramus screws, was the most efficient. The bone screw recommended is the same one developed for extra-alveolar anchorage in the maxilla and mandible (Figure 11).

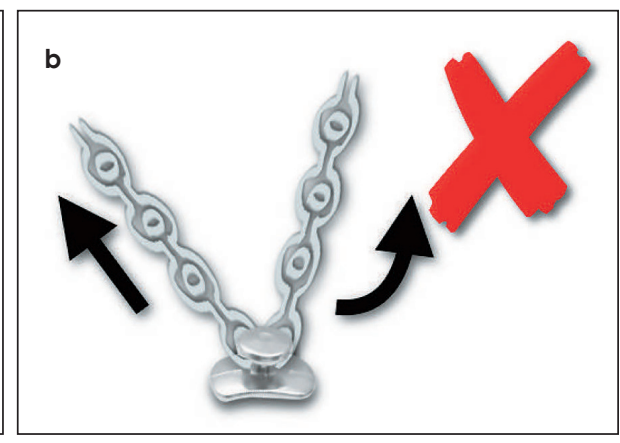

Figure 9(b): Non-safe angle: when elastic chains are attached with an acute angle, there is a high probability that they will slip off the button during normal occlusal function.

\section{Why use $2 \times 14 \mathrm{~mm}$ screws?}

Previous studies have demonstrated that $2 \times 12 \mathrm{~mm}$ extraalveolar screws are appropriate for the mandibular buccal shelf area, because the soft tissue thickness is only about 1-3 mm.10,11 A 2x12 mm screw can usually provide adequate stability and soft tissue clearance when it is inserted in bone about $5 \mathrm{~mm}$. A ramus screw must penetrate thick movable mucosa, as well as the inferior fibers of the temporalis muscle so the $2 \times 14 \mathrm{~mm}$ screw may be needed to provide adequate osseous stability and soft tissue clearance.

Controlling soft tissue inflammation in the anterior ramus area can be a challenge, and it can lead to screw failure. However, a preliminary study found a failure rate of only $5 \%$ for ramus screws, which is lower than for mandibular buccal shelf screws (7.2\%).10,11 Therefore this simple mechanics design is highly recommend to upright impacted second and third molars.

Smooth Mushroom Head For comfort \& retention of elastic chain

Double Neck Design Easy hygiene control \& extra attachment

Stainless Steel High flexibility \& resistance to fracture 4-way Rectangular Holes For lever arm to solve impacted tooth

$14 \mathrm{~mm}$ in Length

Penetrate thicker soft and hard tissue

\section{Sharp Cutting Edge} Easy to penetrate cortical bone, no pre-drilling

Figure 11: A 2x14-mm stainless steel bone screw is designed to be inserted in the ramus as a self-drilling fixture. 


\section{CONCLUSION}

Six impacted molars considerably compromised the occlusal function of a 27yr male. Upper second molars and lower third molars were extracted, bilaterally. Each horizontally impacted second molar was uprighted with a chain of elastics, attached to the distal surface of the tooth and anchored with a ramus bone screw.

\section{OJN}

(Republished with permission from the Author and from International Journal of Orthodontics \& Implantology)

\section{REFERENCES}

1. Aitasalo K, Lehtinen R, Oksala E. An orthopantomographic study of prevalence of impacted teeth. Int J Oral Surg 1972;1:117-20.

2. Catherine KL, Claudia ZYW, Dirk B. Orthodontic uprighting of severely impacted mandibular second molars. Am J Orthod Dentofacial Orthop 2013;143:1 16-24.

3. Bondemark L, Tsiopa J. Prevalence of ectopic eruption, impaction, retention and agenesis of the permanent second molar. Angle Orthod 2007:77:773-778

4. Andreasen JO, Kurol J. The Impacted First and Second Molar. In: Andreasen JO, Petersen JK, Laskin DM, authors. Textbook and Color Atlas of Tooth Impactions. Copenhagen: Munksgaard; 1997. p. 199-208.

5. Camila P, Ana C, Yndira G, Abel C. Failure of eruption of first and second permanent molars. J Clin Pediatr Dent 2003;27(3):239-246.

6. Lin JJ. The wisdom of managing wisdom teeth-Part III. Methods of molar uprighting. Int J Ortho Implantol 201 1;24:4-1 1.

7. Kokich VG, Mathews DA. Impacted Teeth: Surgical and Orthodontic Considerations. Am J Orthod Dentofacial orthop 2011;21:38-49.

8. Hsu YL, Chang CH, Roberts WE. Ortho Bone Screw.The dream screw for next generation's orthodontists. News \& Trends in orthodontics $2011 ; 23: 34-49$.

9. Lee MC, Chang CH. Roberts WE. Compensated, Asymmetric Class II Malocclusion with Horizontal Impaction of Mandibular Second Molars. Int J Ortho Implantol 2014;33:50-62.

10. Chang $\mathrm{CH}$, Roberts WE. A retrospective study of the extra-alveolar screw placement on buccal shelves. Int J Orthod Implantol 2013;32:8089.

11. Chang $\mathrm{CH}$, Roberts WE. Stability of miniscrews on buccal shelves: a retrospective study of 1680 miniscrew insertions by the same orthodontist. Int J Orthod Implantol 2013;30:76-78. 